\title{
Evidence of pertussis clusters in three aged-care facilities in the former Macquarie Area Health Service, NSW
}

\author{
Ala'a Al-Murieb ${ }^{\mathrm{A}}$, Anthony M. Brown ${ }^{\mathrm{A}, \mathrm{B}}$, \\ Alexandra Raulli, Carol George ${ }^{\mathrm{A}}$, \\ Carole Gander $^{\mathrm{A}}$, Patsy Forrester ${ }^{\mathrm{A}}$ \\ and Sharwyn Gibson ${ }^{\mathrm{A}}$
}

\author{
APopulation Health Division, Greater Western Area \\ Health Service \\ ${ }^{\mathrm{B} C o r r e s p o n d i n g ~ a u t h o r . ~ E m a i l: ~ t o n y . b r o w n @ ~}$ \\ gwahs.health.nsw.gov.au
}

\begin{abstract}
During a limited outbreak of pertussis in an area health service in NSW, three clusters occurred in aged-care facilities - the first reported outbreaks of pertussis in Australian nursing homes. The attack rates across the three clusters were $16.7 \%$ for staff and $15.7 \%$ for residents. Our investigation revealed that older adults are not immune to pertussis. We suggest methods for controlling a pertussis outbreak in an aged-care facility.
\end{abstract}

Pertussis is an acute infectious disease that is vaccine preventable but cases still occur because vaccination coverage is not complete and protection wanes with time. Although it is usually regarded as a childhood illness, where severe morbidity and mortality of the disease occur in very young children, pertussis can occur at any age.

Pertussis appears to be endemic in the former Macquarie Area Health Service (now part of Greater Western Area Health Service) in New South Wales (NSW). Epidemics occur in addition to a background of regularly reported cases. We describe here three clusters of pertussis in agedcare facilities (private nursing homes) that occurred as part of an outbreak in 2004. The clusters occurred in two towns within the area health service.

\section{Index case A: aged-care facility 1}

On 8 July 2004, the Macquarie Area Health Service Centre for Population Health was notified of a possible case of pertussis in a staff member of an aged-care facility (facility 1) in a town in the northern part of the area. The staff member had a spasmodic cough and a high serum IgA titre to pertussis (IgA positive). The local general practitioner (GP) managed the person as an incident case. The GP initiated treatment and advised the patient to stay at home. This became index case $\mathrm{A}$.

It was initially thought that the positive blood test of index case A represented a persistent $\operatorname{IgA}$ response to previous pertussis, notified in 2000. As a precaution, the facility was advised to carry out active case finding and appropriate tests. This strategy involved testing all people in the facility with a cough and those who developed a cough in the two to three weeks following identification of index case A. A diagnosis of pertussis was defined as IgA positivity in the presence of a clinically compatible illness.

\section{Index case B: aged-care facilities 2 and 3}

On 9 September 2004, in another town, a case of pertussis (positive IgA) was reported in an assistant in nursing who worked in two aged-care facilities (facility 2 and facility 3 ), and who had had a coughing illness compatible with pertussis for two to three weeks. The date of onset of the illness was between 16 and 23 August (Epiweek 34). The treating GP had started the patient on erythromycin for 14 days and provided a sick leave certificate for five days.

\section{Public health response}

At facility 1, the public health response aimed to ensure that cases and contacts were treated appropriately to limit infection. Symptomatic staff were advised not to attend work until five days of recommended antibiotic treatment had been completed. The facility was advised to implement appropriate infection control guidelines, including: personal hygiene and personal protective equipment; minimising visitors (especially children and pregnant women); and early detection of new cases by testing all staff or residents who developed a cough.

At facility 2 and facility 3 , the public health response was similar. With the permission of the patient, both facilities were informed of the case of pertussis in a casual staff member and were sent fact sheets containing information about the disease. Each facility was actively encouraged to seek new cases among staff and residents, and to test anyone who had a coughing illness. 
Table 1. Cases of pertussis in three aged-care facilities in the former Macquarie Area Health Service (now part of Greater Western Area Health Service) in NSW, 2004

\begin{tabular}{|c|c|c|c|c|c|c|}
\hline & $\begin{array}{l}\text { Number } \\
\text { of staff }\end{array}$ & $\begin{array}{l}\text { Number } \\
\text { of residents }\end{array}$ & $\begin{array}{l}\text { Staff } \\
\text { cases }\end{array}$ & $\begin{array}{l}\text { Resident } \\
\text { cases }\end{array}$ & $\begin{array}{c}\text { Staff attack } \\
\text { rate }(\%)\end{array}$ & $\begin{array}{c}\text { Resident attack } \\
\text { rate (\%) }\end{array}$ \\
\hline Facility 1 & 22 & 24 & 2 & 7 & 9.10 & 29.20 \\
\hline Facility 2 & 90 & 80 & 21 & 15 & 23.30 & 18.80 \\
\hline Facility 3 & 67 & 62 & 7 & 3 & 10.50 & 4.80 \\
\hline Total & 179 & 166 & 30 & 25 & 16.70 & 15.70 \\
\hline
\end{tabular}

\section{Response at facility 1}

Three weeks after the notification of index case A, a further eight cases were reported from facility 1 . A number of residents and staff had developed a cough and had had blood tests. Seven out of 24 residents and one additional staff member were IgA positive and reported to have a cough (Table 1).

Over the next week, other concerned asymptomatic staff members arranged to be tested and four more asymptomatic staff were found to be IgA positive. Being asymptomatic, these people were not regarded as cases.

\section{Response at facility 2}

Facility 2 actively identified and tested residents with a cough. A number of IgA positive tests among the residents were reported on 15 September 2004 (Table 1).

In response, an investigation team visited facility 2 to obtain epidemiological information about the people who tested positive in order to support the facility and advise on appropriate infection-control procedures. Detailed information about the cases was difficult to obtain as age, dementia and illness meant that most of the people could not remember or communicate their immunisation status or could not remember having previously had a whooping cough-like illness.

The team recommended that all residents who had developed a cough in the preceding three weeks should receive antibiotic treatment regardless of IgA status. All residents who developed a cough in the following week were treated empirically (i.e. treatment was initiated before a diagnosis of pertussis was confirmed).

Symptomatic staff were advised to be tested, to be treated and to take leave from work until they had completed five days of antibiotic treatment. A fact sheet was distributed to visitors, and signs were posted to exclude high-risk category visitors (children less than one year of age, children between 12 months and 5 years who were not fully immunised and women in the last month of pregnancy). This outbreak and the infection control procedures generated local media interest and a media release was issued and followed up with television and radio interviews.

\section{Response at facility 3}

Despite having received the same information and advice as facility 2 , facility 3 did not actively identify residents and staff with a cough. Facility 3 had fewer symptomatic residents and staff. A few people developed a cough in the following weeks and tested positive (Table 1).

\section{Results}

The number of staff and residents found to have pertussis and the attack rates in each of the three aged-care facilities are shown in Table 1. In each facility, a significant proportion of the staff was affected. Facility 2 and facility 3 were situated in the same town. In both facilities, a significantly larger proportion of staff were affected than residents.

A review of the notifications for pertussis in facility 2 and facility 3 , and a calculation of their onset dates, revealed five cases with onset before index case B.

\section{Discussion}

These three clusters arose during 2004, a high incidence year for pertussis in the former Macquarie Area Health Service. The clusters occurred in two different towns in the area: facility 2 and facility 3 were situated in the same town and had a significantly larger proportion of staff affected than residents.

Pertussis has generally been considered a disease of children. In NSW, there has been a shift in the age distribution of new cases of pertussis from pre-teens to adolescents. ${ }^{1}$ Pertussis is not assumed to be significant in adults. Our data clearly show that outbreaks of pertussis can occur in elderly people. Other researchers have found an incidence rate of serologically defined infection of 19.7 per 100 person-years in a group of 100 people aged over $65 .^{2}$ Pertussis in adults produces considerable morbidity and, in addition, provides a significant reservoir of infection for children.

Outbreaks of infectious diseases such as influenza are well known to occur in nursing homes. ${ }^{3,4}$ Pertussis outbreaks have less commonly been reported: an outbreak of pertussis in a Wisconsin nursing home in the 1980s and an outbreak in severely handicapped patients in a neurological ward have previously been described. ${ }^{5,6}$ This occurrence 


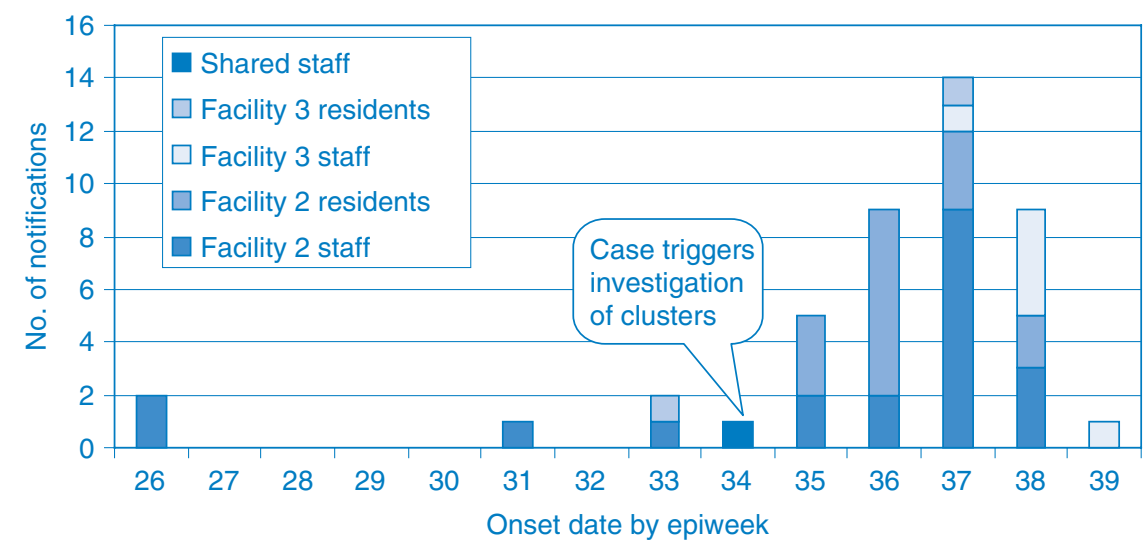

Figure 1. Week of onset of pertussis cases in aged-care facilities 2 and 3 in the former Macquarie Area Health Service (now part of Greater Western Area Health Service) in NSW, 2004. Source: NSW Health Notifiable Disease Database.

is the first reported in an Australian nursing home. In 2005, the United States' Centers for Disease Control and Prevention (CDC) reported outbreaks of pertussis in hospitals in three states. ${ }^{7}$

Importantly, outbreaks of pertussis in residential institutions have also involved staff. In an investigation of pertussis in a residential facility for handicapped people, the epidemiological evidence suggested that the infection was introduced and spread by staff. ${ }^{8}$ It is common for staff to work in more than one aged-care facility and this movement facilitates disease transmission. In facility 2 and facility 3 , the review of notifications of pertussis and the calculation of their onset dates revealed five cases in these two facilities reported before index case B (Figure 1). Because staff members had pertussis before the index case triggered investigation, it is most likely that the infection was introduced to the facilities by staff. As pertussis is endemic in this area, undiagnosed cases maintain the disease in the community and may be responsible for outbreaks.

The outbreak was controlled by rapid identification and treatment of people with a cough. The implementation of prevention strategies also played an important role in the control of the outbreak. Although it has been reported that prophylactic antibiotics have a role in controlling the extent of pertussis outbreaks, mass prophylactic antibiotics were not used in this situation as they are not recommended by NSW Health guidelines. ${ }^{9,10}$ Hospital outbreaks reported by CDC did use prophylactic antibiotics for contacts and, although further cases did not occur, the role of prophylactic antibiotics remains uncertain. ${ }^{7}$

Vaccination is the mainstay of pertussis prevention although one report indicated that the attack rate in immunised residents was the same as for non-immunised residents. ${ }^{8}$ Until recently, there has not been a pertussis vaccine recommended for use in adults: normally, immunisation of this age group would have occurred many years earlier in childhood and immunity may have waned. The efficacy of pertussis vaccination in adults during an outbreak has been suggested but has yet to be established. 2,7,8

These three clusters also underline the limitation of detection using specific IgA alone. IgA may persist for some time after the acute infection and may have delayed the identification of the initial case. ${ }^{11}$ However, IgA may also be positive without symptoms and a diagnosis of pertussis depends on IgA positivity in the presence of a clinically compatible illness. In facility 2 , a number of asymptomatic staff were tested and found to be positive for IgA.

\section{Conclusion}

This investigation has shown that pertussis outbreaks can occur in adults in semi-enclosed communities, such as nursing homes, and that staff can readily transmit the disease. With the present availability of a multivalent vaccine containing pertussis that is suitable for adults, routine vaccination of staff at aged-care facilities should be considered.

\section{References}

1. Brotherton J, McAnulty J. A Pertussis epidemic in NSW: How epidemiology reflects vaccination policy. $N S W$ Public Health Bull 2003; 14: 77-81. doi:10.1071/NB03024

2. Hodder SL, Cherry JD, Mortimer EA, Ford AB, Gornbein J, Papp K. Antibody responses to Bordetella pertussis antigens and clinical correlations in elderly community residents. Clin Infect Dis 2000; 31(1): 7-14. doi:10.1086/313913

3. Guy RJ, Di Natale R, Kelly H, Lambert SB, Tobin S, Robinson $\mathrm{PM}$ et al. Influenza outbreaks in aged-care facilities: staff vaccination and the emerging use of antiviral therapy. Med $J$ Aust 2004; 180(12): 640-2.

4. Bush KA, McAnulty J, McPhie K, Reynolds R, Boomer M, Clarkson LM et al. Southern New South Wales Public Health Unit. Antiviral prophylaxis in the management of an influenza outbreak in an aged care facility. Commun Dis Intell 2004; 28(3): 396-400. 
5. Addiss DG, Davis JP, Meade BD, Burstyn DG, Meissner M, Zastrow JA et al. A pertussis outbreak in a Wisconsin nursing home. J Infect Dis 1991; 164(4): 704-10.

6. Tomoda T, Ogura H, Kurashige T. The longevity of the immune response to filamentous hemagglutinin and pertussis toxin in patients with pertussis in a semiclosed community. J Infect Dis 1992; 166(4): 908-10.

7. Centers for Disease Control and Prevention. Outbreaks of pertussis associated with hospitals: Kentucky, Pennsylvania and Oregon, 2003. Morb Mortal Wkly Rep 2005; 54: 67-71.

8. Tanaka Y, Fujinaga K, Goto A, Iwai H, Aoyama T, Murase Y et al. Outbreak of pertussis in a residential facility for handicapped people. Dev Biol Stand 1991; 73: 329-32.
9. Partiarca PA, Steketee RW, Biellik RJ, Wassilak SG, Brink EW, Burstyn DG et al. Outbreaks of pertussis in the United States: the Wisconsin experience. Tokai J Exp Clin Med 1988; 13(Suppl.): 117-23.

10. NSW Health. Occupational screening and vaccination against infectious diseases. NSW Health Circular 2003/91. NSW Health: Sydney; 2003.

11. Heininger U, Cherry JD, Stehr K. Serologic response and antibody-titer decay in adults with pertussis. Clin Infect Dis 2004; 38(4): 591-4. doi:10.1086/381439 